\title{
Pulsars in gamma rays: What Fermi is teaching us
}

\author{
Matthew Kerr ${ }^{1}$ and the Fermi-LAT Collaboration \\ ${ }^{1}$ Kavli Institute for Particle Astrophysics and Cosmology, Stanford University, \\ Stanford, CA 94305, USA \\ email: kerrm@stanford.edu
}

\begin{abstract}
The 2nd Fermi-LAT pulsar catalog includes $117 \gamma$-ray pulsars, of which roughly one third are millisecond pulsars (MSPs) while the remaining two thirds split evenly into young radio-loud and radio-quiet pulsars. Although this large population will enable future, detailed studies of emission mechanisms and the evolution of the underlying neutron star population, some nearly-universal properties are already clear and unequivocal. We discuss some of these aspects below, including the altitude of the $\gamma$-ray emission site, the shape of the $\gamma$-ray spectrum, and the implications of the latter for the radiation mechanism.
\end{abstract}

Keywords. pulsar: general, gamma rays: observations, radiation mechanisms: nonthermal

\section{Introduction}

Since their discovery forty-five years ago (Hewish et al. 1968), pulsars have primarily been studied at radio wavelengths - a natural consequence of their discovery mode and the progression to ever larger antennae and higher-bandwidth receivers. Yet pulsars emit only a tiny fraction $\left(\sim 10^{-5}\right)$ of their spindown luminosity $\dot{E}$ at radio wavelengths. Furthermore, their high brightness temperature and variability on timescales as short as a few ns (Hankins et al. 2003) indicate a coherent emission process from a small volume. Making inferences about the global structure of the pulsar magnetosphere from such local measurements is difficult.

Young pulsars and MSPs, however, are extraordinarily efficient sources of $\gamma$ rays, converting $1-100 \%$ of their spindown luminosity into $100 \mathrm{MeV}-30 \mathrm{GeV}$ emission (e.g. Abdo et al. $2010 \mathrm{~b}$, and noting that efficiencies can be highly uncertain due to anisotropic beams and distance uncertainty). The emission is incoherent, and such efficiency implies much of the potential induced by the pulsar's magnetic field is involved in particle acceleration, i.e. the acceleration and emitting volumes are large on the scale of the magnetosphere. Moreover, the ultrarelativistic particles powering the $\gamma$ emission propagate along magnetic field lines and beam their emission along them. Thus, $\gamma$ rays are a tracer of the large scale magnetic field structure.

Prior to the Fermi Large Area Telescope (LAT), only a handful of $\gamma$-ray pulsars were known, primarily of the young, radio-loud class, limiting their usefulness in studies of the magnetosphere and the underlying pulsar population. The situation evolved rapidly after the successful launch of Fermi, with the discovery of $\gamma$-ray emission from MSPs (Abdo et al. 2009a) and radio-quiet young pulsars from fecund "blind" searches of the LAT data (Abdo et al. 2009b). The first Fermi pulsar catalog (Abdo et al. 2010b) provided properties of $46 \gamma$-ray pulsars, an appreciable increase in number and diversity. The second Fermi pulsar catalog (2PC), in preparation, includes $117 \gamma$-ray pulsars and more sophisticated analysis. For instance, light curves use photon weights (Kerr 2011) which 
provide increased $\mathrm{S} / \mathrm{N}$ and a reliably-estimated background level. In-depth spectral analyses attempt to disentangle magnetospheric emission from astrophysical backgrounds, including pulsar wind nebulae.

2PC will undoubtedly fuel detailed population (synthesis) studies. The breadth and depth of the observed population, however, suggest that any observed trends are nighuniversal properties of $\gamma$-ray pulsars and are worth studying in their own light. Below, we detail two such trends. First, $\gamma$-ray emission appears to originate in the outer magnetosphere. Second, the spectral properties of the emission, for all pulsars save the Crab, are consistent with curvature radiation.

\section{2. $\gamma$-ray emission altitude}

The height in the magnetosphere from which the bulk of $\gamma$-ray emission arises is probed through several mechanisms. The first, relatively model-independent method, depends on the altitude-dependent opacity for pair production by $\gamma$-rays on the strong magnetic field near the surface of the neutron star. Two additional methods depend on the assumed structure of the magnetic field, but with little sensitivity to the details. Below, we briefly discuss each method.

\subsection{Magnetic opacity}

In strong magnetic fields, classically forbidden processes, such as one-photon pair production and annihilation, become allowed (e.g. Harding 1991). The former process, in particular, attenuates high-energy $\gamma$ rays. The altitude by which the magnetic field has weakened sufficiently to allow photons of energy $E$ to escape is given by $r \approx$ $\left(B_{12} E / 1.76 \mathrm{GeV}\right)^{2 / 7} P^{1 / 7} R_{\star}$ (Baring 2004) with $B_{12}$ the magnetic field in units of $10^{12} \mathrm{G}$ and $R_{\star}$ the neutron star radius. The observation of photons well above $10 \mathrm{GeV}$ for young neutron stars implies production above a few $R_{\star}$. Although this constraint is small in terms of the distance to the light cylinder, $R_{L C}$ (which depends on period, but for young

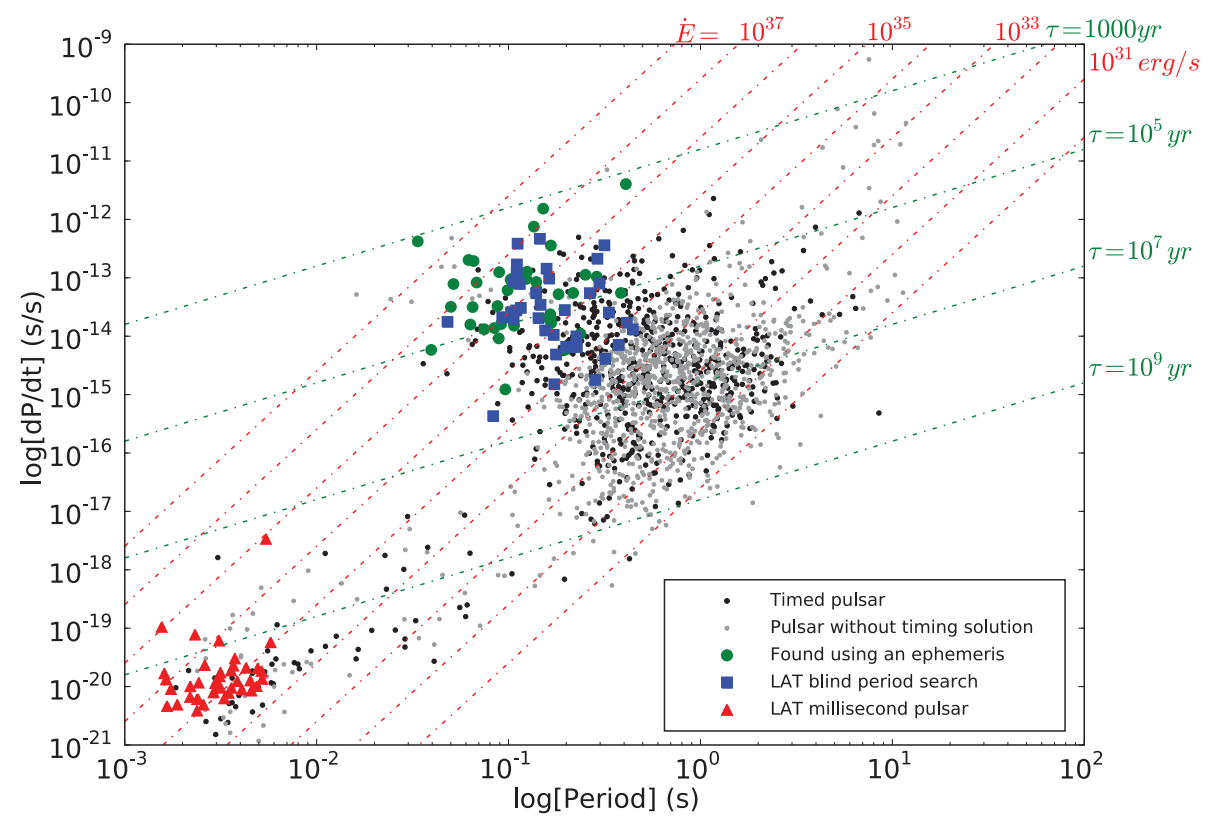

Figure 1. The period $(\mathrm{P})$ and period derivative $(\mathrm{dP} / \mathrm{dt})$ of $2 \mathrm{PC}$ pulsars. 
pulsars is $\sim 200-2000 R_{\star}$ ), it rules out emission from particles accelerated near the polar cap (e.g. Arons and Scharlemann 1979).

Additional evidence along these lines stems from the shape of the spectral cutoff observed in Fermi pulsars. Since the opacity to magnetic attenuation depends on energy, the curvature radiation spectrum from low-altitude photons is suppressed superexponentially. The observed spectra, however, show no such suppression. Indeed, as discussed below, they typically decay subexponentially; see, e.g. the spectra of Geminga (Abdo et al. 2010a) and Vela (Figure 3).

\subsection{Caustics}

Although observations of high energy $(\gg 1 \mathrm{GeV}$ ) photons and gradual spectral cutoffs rule out emission from near the stellar surface, they do not help to distinguish between "low altitude" (10-30\% of $\left.R_{L C}\right)$ and "outer magnetospheric" (OM; $\left.>30 \% R_{L C}\right)$ origin. These definitions are somewhat arbitrary, and another convenient division between low altitude and $\mathrm{OM}$ emission is the null charge (NC) surface, where the magnetic field projected along the spin axis vanishes. Indeed, the NC surface is the traditional starward edge of the eponymous "outer gap" of such particle acceleration models (e.g. Cheng, Ho, and Ruderman 1986). Other models, such as the slot gap (e.g. Muslimov and Harding 2003), predict emission both above and below the NC surface.

A general property of emission from the $\mathrm{OM}$ is the formation of caustics from near cancellation of relativistic aberration, time of flight across the magnetosphere, and field line sweepback (Morini 1983). The degree of sweepback determines the precise location in the magnetosphere for caustic formation, but static, vacuum, and force-free MHD magnetic field solutions all demonstrate such features, as shown e.g. by Romani and Yadigaroglu (1995), Watters et al. (2009), and Bai and Spitkovsky (2010), respectively. In the population of young $2 \mathrm{PC}$ pulsars, caustic-like peaks are a nearly universal light curve feature, a clear indication of $\mathrm{OM}$ origin.

For radio-loud pulsars, two important quantities may be estimated: $\delta$, the phase lag from the magnetic axis to the first $\gamma$ peak, and $\Delta$, the separation of (if present) the two primary $\gamma$ peaks. Generally, OM magnetosphere and emission models predict a correlation of these two quantities, with the shape depending on the model details. The observed correlation of 1PC agrees roughly with "outer gap" models (see e.g., Watters et al. 2009) and the correlation is strengthened for young pulsars in 2PC. The precise phase relation is a sensitive probe of the structure of the magnetosphere and may reveal, e.g., the extent to which currents shape the magnetosphere. MSPs show a similar, though more scattered, correlation, with some exceptions. These are "aligned" MSPs (e.g. PSR B1937+21, see Guillemot et al. 2012) whose $\gamma$-ray and radio emission occur at the same rotational phase, inviting speculation that both beams originate from the same OM caustics.

\subsection{Unpulsed magnetospheric emission}

A final argument for the dominance of OM $\gamma$-ray emission is the general absence of unpulsed emission - that is, emission present throughout all rotational phases. The level of such emission is robustly estimated in $2 \mathrm{PC}$ through the use of photon weights; these weights are derived directly from the phase-averaged spectral model, which accounts for the spatial distribution of astrophysical backgrounds. In contrast, background levels in $1 \mathrm{PC}$ were estimated by considering photon counts in an off-pulse annulus around the pulsar, subject to substantial inaccuracy from spatial variation in the background level.

In the altitude range above the polar cap but below the outer magnetosphere, the divergence of field lines causes beams from the open zone to illuminate an ever greater fraction of the sky with increasing altitude, leading to emission with little modulation at 
sufficiently high altitudes. At even higher (OM) altitudes, caustics begin to form, leading to anisotropic and fully-pulsed emission. Thus, the presence of unpulsed magnetospheric emission is a sensitive indicator of emission from low altitudes.

In the 2PC, only two pulsars (PSRs J1836+5926 and J2021+4026) have levels of unpulsed emission competitive with or exceeding modulated emission, while the remaining pulsars show subdominant or absent off-peak emission, suggesting that emission from low altitude plays a minimal role in most $\gamma$-ray pulsars.

\section{3. $\gamma$-ray emission mechanism}

A second question $2 \mathrm{PC}$ is poised to answer is the nature of the dominant $\gamma$-ray radiation mechanism. The simplest, and arguably most widely favored, process for producing the $\mathrm{GeV}$ photons is curvature radiation from leptons with Lorentz factors of $\sim 10^{7}$ flowing along magnetic field lines. The primary alternative is inverse Compton emission, potentially from synchrotron seed photons (synchrotron/self-Compton). A detailed review of these models is beyond the scope of this paper.

The primary discriminator between the two mechanisms is the spectral shape of the broadband emission. While a few bright pulsars are detected in hard X-rays and soft $\gamma$ rays, the LAT GeV spectrum is the primary observable. Although the expected spectral shape is a complicated function of the acceleration and cooling of the emitting particles, the spectral cutoff for curvature radiation is cleanly determined by the highest-energy particles, and the absence of a strong cutoff could be interpreted as evidence for IC emission. However, as we show below, two effects - one technical and one physicalconfound such simple conclusions, and the vast majority of Fermi pulsar emission is consistent with curvature radiation.

\subsection{Monoenergetic spectrum}

The curvature radiation spectrum of a single particle is set by the particle energy and the radius of curvature of the magnetic field. This monoenergetic photon spectrum, summed over polarizations, takes the simple form (Jackson 1998)

$$
\frac{d N}{d E} \propto \int_{E / E_{c}}^{\infty} K_{5 / 3}(x) d x,
$$

where $K$ is a modified Bessel function. In the limits $E \ll E_{c}$ and $E \gg E_{c}$, the spectrum asymptotes to the well known forms $d N / d E \propto E^{-2 / 3}$ and $d N / d E \propto E^{-1 / 2} \exp -E / E_{c}$, respectively. Typical measured and expected values of $E_{c}$ of $1-3 \mathrm{GeV}$ fall squarely in the middle of the LAT passband. The functional form most often used to fit LAT spectra, $d N / d E \propto E^{-\Gamma} \exp -E / E_{c}$ (PLEC), can only mimic one or the other of these two limits. Thus one must be cautious in interpreting a failure of the PLEC model to fully describe the data as a failure for the curvature radiation mechanism.

We briefly probe the magnitude of the error made in approximating a monoenergetic curvature spectrum with the PLEC model. In Figure 2 (left), we show the monoenergetic photon spectrum with $E_{c}=2 \mathrm{GeV}$. The PLEC spectrum that most closely agrees at energies $E<E_{c}$ with the exact curvature spectrum has $\Gamma=0.7$, close to the expected asymptotic value $2 / 3$. However, this photon index is softer than the high-energy value of $1 / 2$, causing the PLEC model to appreciably underestimate the monoenergetic spectrum for $E>E_{c}$, resulting in a discrepancy of $\sim 20 \%$ at $20 \mathrm{GeV}$ (Figure 2, right). Such energies are well within reach of the LAT, and indeed the observed spectra of bright pulsars such as Vela show a disagreement of just such a magnitude. These conclusions are in line with 

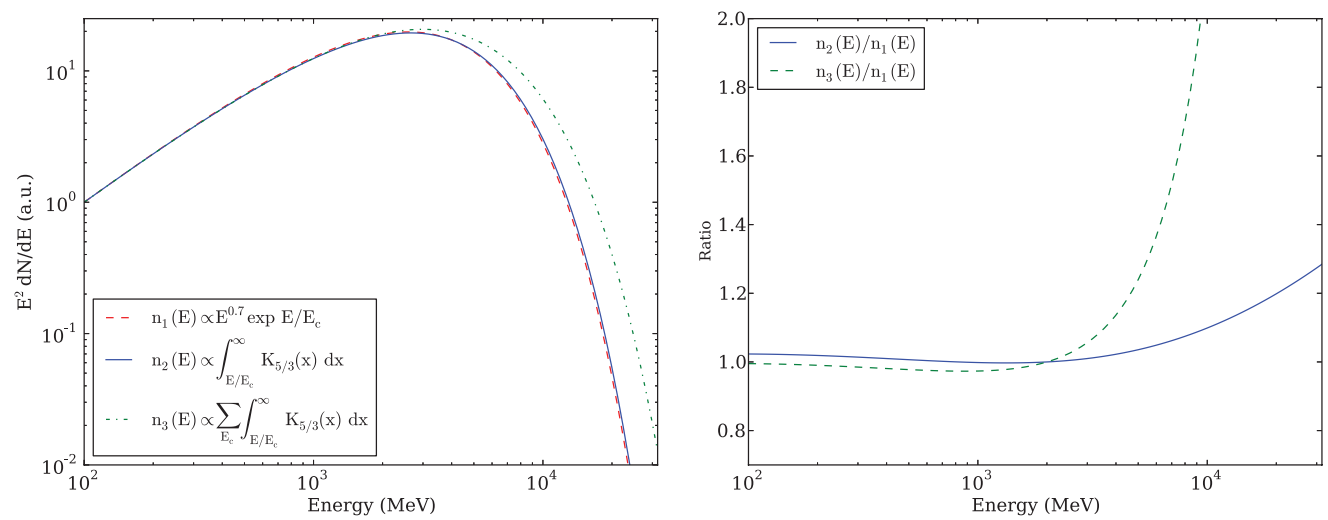

Figure 2. Left: A monoenergetic $\left(E_{c}=2 \mathrm{GeV}\right)$ curvature radiation spectrum is shown in solid blue, along with a PLEC model that agrees closely with it below $E_{c}$ (dashed red). A mixture of monoenergetic spectra appears in dot-dashed green. Right: The ratio of monoenergetic spectra to a PLEC approximation. A single spectrum $\left(E_{c}=2 \mathrm{GeV}\right)$ appears in solid blue, while the mixture spectrum described in the text is shown in dashed green.

those of the phase-resolved spectroscopy of Abdo et al. (2010c) where spectra in narrow phase windows agree well with a PLEC model.

\subsection{Spectral superpositions}

An even more drastic effect stems from the caustic nature of OM emission. Because emission piles up over a large volume of the magnetosphere, multiple field lines / radii of curvature are expected to contribute to the phase-averaged spectrum. For a constant accelerating electric field, this leads to a linear spread in $E_{c}$. In Figure 2 (left), we show a mixture of 10 spectra with $E_{c}$ uniformly distributed from 1 to $3 \mathrm{GeV}$. The low energy (approximately power law) portion of the spectrum is essentially unchanged from a monoenergetic spectrum with the mean $E_{c}=2 \mathrm{GeV}$, while the portion above $2 \mathrm{GeV}$ is much harder than the single $E_{c}=2 \mathrm{GeV}$ spectrum. Figure 2 shows that in the case

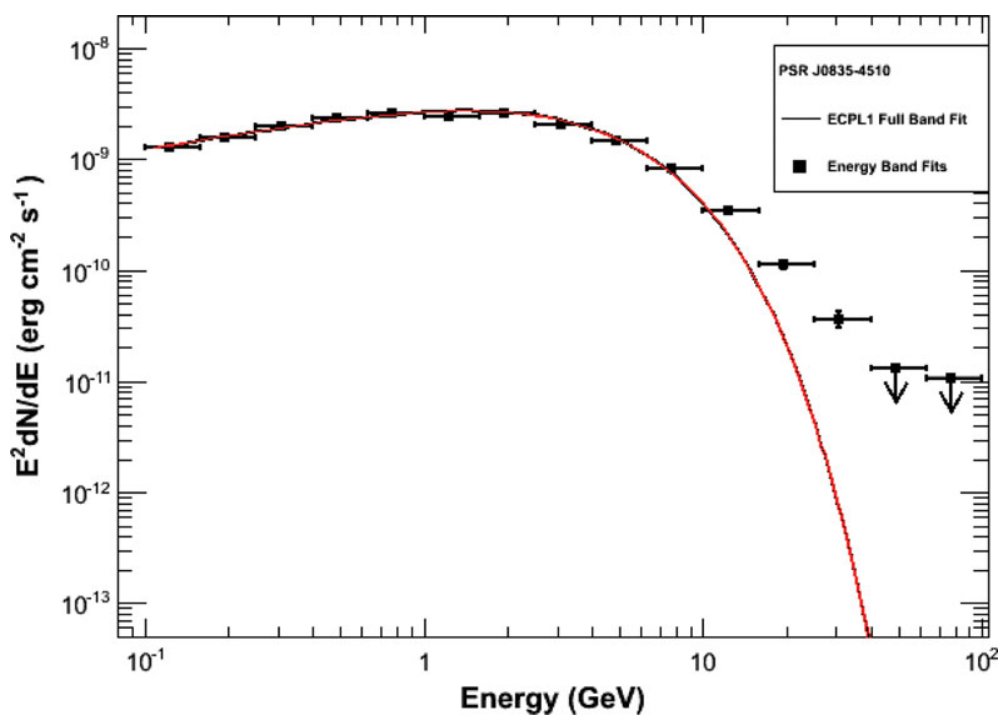

Figure 3. The 2PC phase-averaged spectrum of PSR J0835-4510 (Vela). The best fit PLEC is shown as the solid, red line and substantially underestimates the observed spectrum. 
of this modest mixture the simple PLEC model can underestimate the spectrum above $10 \mathrm{GeV}$ by an order of magnitude at the highest energies accessible to the LAT.

\section{Conclusions and the future}

In summary, the dramatically increased size and breadth of the $\gamma$-ray pulsar population is finally settling some of the longstanding questions about the origin and mechanism of $\gamma$ ray emission. We now know that $\gamma$ rays arise primarily from the outer magnetosphere, and that the spectral signature is consistent with electrons and positrons emitting curvature radiation. Moreover, because $\gamma$ rays are such an excellent tracer of the magnetosphere, detailed analysis of the Fermi light curves and spectra offer the opportunity to test more sophisticated emission theories and probe the magnetosphere structure in modelindependent ways.

Although the trends discussed above are nearly universal, the few exceptions also offer tantalizing opportunities for better understanding of the pulsar machine. The recent detections by VERITAS and MAGIC (e.g. Aliu et al. 2011, Aleksić et al. 2011) of pulsed photons through energies $>100 \mathrm{GeV}$ from the Crab pulsar imply at least some of the Crab's $\gamma$-ray emission originates from inverse Compton scattering (see e.g. Lyutikov, Otte, and McCann 2012). Indeed, of all pulsars detected above $100 \mathrm{MeV}$, only the Crab peaks in $\nu F_{\nu}$ well below $1 \mathrm{MeV}$, indicating the presence of a strong synchrotron component and motivating a synchrotron/self-Compton (SSC) picture for $\gamma$-ray emission. Discovery and analysis of "transition" objects such as PSR B1509-58, whose $\nu F_{\nu}$ peak is of order $1 \mathrm{MeV}$ (Kuiper et al. 1999, Pilia et al. 2010, den Hartog et al. in prep), may shed light on the transition, if any, from a SSC-dominated radiation mechanism to a curvature-dominated one.

\section{References}

Abdo, A. A., et al. 2009a, Science, 325, 848

-. 2009b, Science, 325, 840

-. 2010a, ApJ, 720, 272

-. 2010b, ApJS, 187, 460

-. 2010c, ApJ, 713, 154

Aleksić, J., et al. 2011, ApJ, 742, 43

Aliu, E., et al. 2011, Science, 334, 69

Arons, J. \& Scharlemann, E. T. 1979, ApJ, 231, 854

Bai, X.-N. \& Spitkovsky, A. 2010, ApJ, 715, 1282

Baring, M. G. 2004, Advances in Space Research, 33, 552

Guillemot, L., et al. 2012, ApJ, 744, 33

Hankins, T. H., Kern, J. S., Weatherall, J. C., \& Eilek, J. A. 2003, Nature, 422, 141

Harding, A. K. 1991, Science, 251, 1033

Hewish, A., Bell, S. J., Pilkington, J. D. H., et al. 1968, Nature, 217, 709

Jackson, J. D. 1998, Classical Electrodynamics, 3rd Edition

Kerr, M. 2011, ApJ, 732, 38

Kuiper, L., Hermsen, W., Krijger, J. M., et al. 1999, A\&\&A, 351, 119

Lyutikov, M., Otte, N., \& McCann, A. 2012, ApJ, 754, 33

Morini, M. 1983, MNRAS, 202, 495

Muslimov, A. G. \& Harding, A. K. 2003, ApJ, 588, 430

Pilia, M., et al. 2010, ApJ, 723, 707

Romani, R. W. \& Yadigaroglu, I.-A. 1995, ApJ, 438, 314

Watters, K. P., Romani, R. W., Weltevrede, P., \& Johnston, S. 2009, ApJ, 695, 1289 